\title{
Innovative Applications of Raman Microscopy
}

\author{
Peng Wang ${ }^{1}$, Juergen Sawatzki ${ }^{2}$ and Thomas J. Tague $\mathrm{Jr}^{3}$ \\ ${ }^{1,3}$ Bruker Optics Inc., Billerica, MA, USA \\ ${ }^{2}$ Bruker Optik GmbH, Ettlingen, Germany
}

Raman spectroscopy is one of the most commonly used techniques for materials identification and characterization. By measuring vibrational as well as lattice and other low frequency modes in a system, it provides unique information for molecular compositions as well as backbone and crystalline structures. Therefore, Raman spectroscopy finds extensive application to various fields including but not limited to pharmaceuticals, polymers, forensics, cultural heritage, life science, mineralogy, materials science, and more. State-of-the-art Raman microscopy enhances the investigation of materials by providing non-invasive microscopic analysis featuring minimal sample preparation, diffraction limited lateral resolution and subsurface accessibility due to its confocal design. It can not only be used to analyze pure compounds but also be applied to mixture analysis, composite structure analysis, orientation and crystallinity analysis and so on. In this paper, some recent applications of Raman microscopy are presented and discussed.

A Bruker Senterra dispersive Raman microscope was used for all of the measurements. As shown in Figure 1, Senterra is built on the Olympus BX51 research grade optical microscopy platform. It enables the use of the full range of optical microscopy tools including aperture stop, dark field illumination, polarized light observation, Nomarski DIC enhancement, and fluorescence illumination to allow the user to achieve optimal visualization of the sample. Senterra accommodates up to 4 excitation lasers ranging from $488 \mathrm{~nm}$ to $1064 \mathrm{~nm}$, significantly increasing research capabilities. The system performs automatic and continuous wavelength calibrations to provide wavelength accuracy of at least $0.1 \mathrm{~cm}^{-1} \mathrm{RMS}$. Ondemand confocal or high throughput measurements are made possible by utilizing a computer controlled array of pinholes and slits. For Raman mappings, Senterra is equipped with a high performance automatic stage with $0.1 \mu \mathrm{m}$ positioning accuracy which allows very precise mapping based imaging measurements.

Alkali-silica reaction (ASR) is one of the mechanisms responsible for the degradation process resulting in cracking of concrete structures. This reaction forms a swelling gel of calcium silicate hydrate (CSH), which increases in volume in the presence of water, causing spalling and loss of strength of the concrete, finally leading to its failure. In this study, Raman line mapping was conducted on a partially degraded concrete material using the $785 \mathrm{~nm}$ laser. An intact CSH phase was located in between the degraded area and a stone of the concrete matrix as shown in Figure 2.

Raman mapping based imaging was used to study mayonnaise with the 532nm laser. 3 major components are identified. Individual spectra and the corresponding chemical image showing the distribution are shown in Figure 3. Such chemical imaging offers great potential for food formulation, safety and quality control research.

Raman microscopy has unique applications in analyzing small samples and multicomponent systems. Fast and noninvasive measurements make it a powerful tool for many fields of interest. It not only 
provides chemical information, but also associates the information with real two or even three dimensional structure.
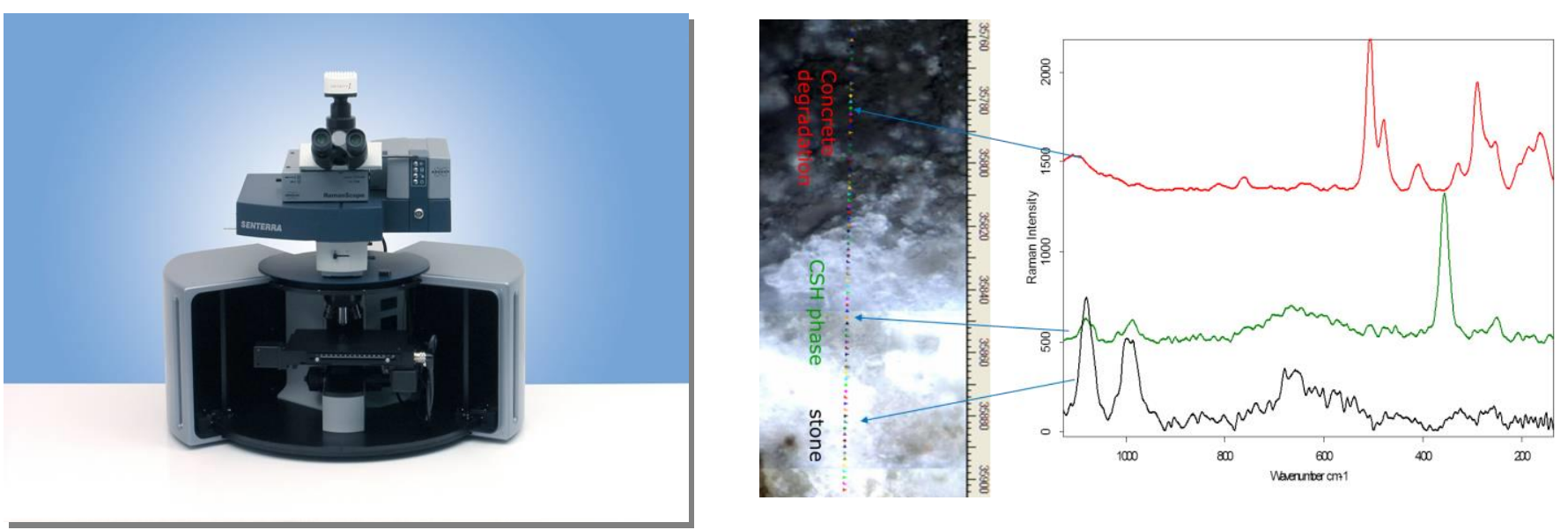

Figure 1. Senterra Raman Microscope

Figure 2. Raman line mapping on degraded concrete.

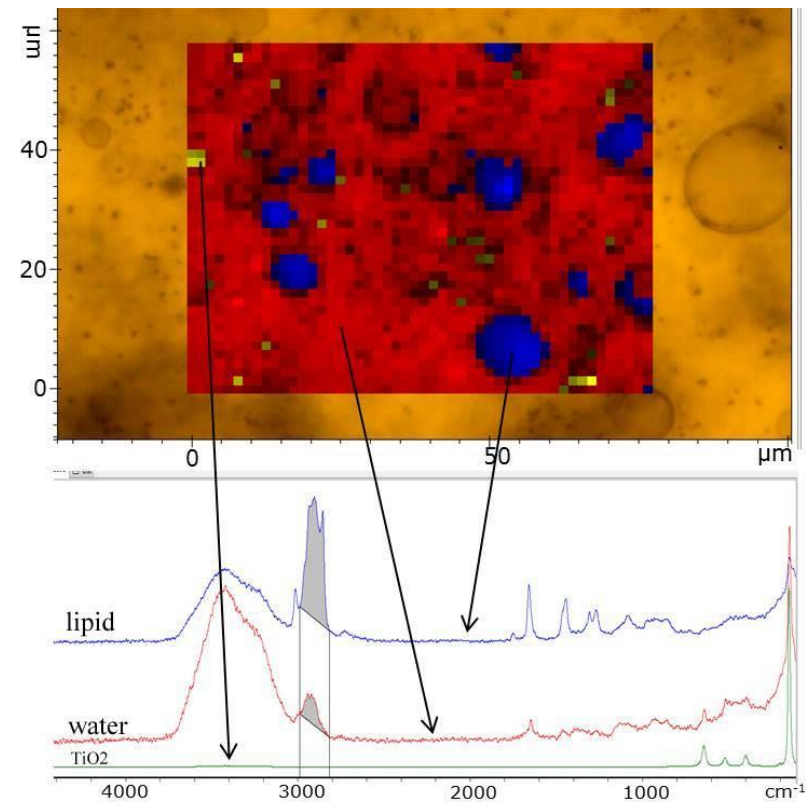

Figure 3. Raman mapping of mayonnaise. 